\title{
Diseño de interfaz gráfica para analizar el ciclo de marcha del cuerpo humano en software libre
}

\section{Design of a graphical user interface to analyze the gait cycle of the human body using free software}

CARRILLO-HERNÁNDEZ, Didia†*, TERRONES-LARA, Yered Uriel, GARCÍA-CERVANTES, Heraclio y BLANCO-MIRANDA, Alan David

Universidad Tecnológica de León, Cuerpo Académico de Bioingeniería

ID $1^{\text {er }}$ Autor: Didia, Carrillo-Hernández / ORC ID: 0000-0001-9989-5884, Researcher ID Thomson: ABF-4839-2020, CVU CONACYT ID: 936937

ID $1^{\mathrm{er}}$ Coautor: Yered Uriel, Terrones-Lara / ORC ID: 0000-0001-7828-8700, Researcher ID Thomson: ABF-5110-2020 CVU CONACYT ID: 1095817

ID $2^{\text {do }}$ Coautor: Heraclio, García-Cervantes / ORC ID: 0000-0002-4229-9229, Researcher ID Thomson: X-5622-2019, CVU CONACYT ID: 290829

ID $3^{\text {er }}$ Coautor: Alan David, Blanco-Miranda / ORC ID: 0000-0002-8595-8634, Researcher ID Thomson: W-9701-2019, CVU CONACYT ID: 298274

DOI: $10.35429 / J B E B .2020 .12 .4 .13 .17$

Recibido 12 de Septiembre, 2020; Aceptado 30 Diciembre, 2020

Resumen

Actualmente en el país existen mas de 27 mil casos de amputaciones anuales y más del $80 \%$ corresponden a miembros inferiores, por lo tanto, la demanda de equipos protésicos es mayor a lo que las instituciones de sector salud pueden otorgar. Cabe destacar que los equipos desarrollados por dichas instituciones solo son equipos pasivos, por lo que solo el $10 \%$ de los pacientes que reciben un equipo protésico termina satisfactoriamente su rehabilitación. Los principales problemas que enfrenta el paciente frente a la adaptación con su equipo protésico, es el tiempo de respuesta y alineación vs el miembro sano, ya que no cuenta con un sistema de control inteligente que le permita responder en tiempo real como lo hacia el miembro perdido. Esto provoca desfaces al realizar su ciclo de marcha, esto con el transcurso del tiempo puede traer consigo anomalías en su postura repercutiendo en la alineación de su sistema motriz. El presente trabajo permite analizar el rango de movimiento de tobillos y rodillas, además determina la velocidad angular de ambos, es información fundamental para el desarrollo de sistemas de control necesarios para equipos protésicos activos. El lenguaje de progamación donde fue desarrollada es el software Python 3.7 y adicional reproducir la simulación del ciclo de marcha.

Animación 3D, Cinemática, Miembros inferiores

\begin{abstract}
Currently in the country there are more than 27 thousand cases of annual amputations and more than $80 \%$ correspond to lower limbs, therefore, the demand for prosthetic equipment is greater than what the health sector institutions can provide. It should be noted that the equipment developed by these institutions is only passive equipment, so that only $10 \%$ of patients who receive a prosthetic equipment successfully complete their rehabilitation. The main problems that the patient faces when adapting to their prosthetic equipment is the response time and alignment vs the healthy limb, since it does not have an intelligent control system that allows them to respond in real time as the losted limb did. This causes gaps when performing your gait cycle, this over time can bring about abnormalities in your posture affecting the alignment of your motor system. This work allows us to analyze the range of motion of the ankles and knees, in addition to determining the angular velocity of both, it is essential information for the development of control systems necessary for active prosthetic equipment. The programming language where it was developed is the Python 3.7 software and additionally reproduce the simulation of the gait cycle.
\end{abstract}

3d Animation, Cinematics, Lower Limbs

Citación: CARRILLO-HERNÁNDEZ, Didia, TERRONES-LARA, Yered Uriel, GARCÍA-CERVANTES, Heraclio y BLANCO-MIRANDA, Alan David. Diseño de interfaz gráfica para analizar el ciclo de marcha del cuerpo humano en software libre. Revista de Ingeniería Biomédica y Biotecnología. 2020. 4-12: 13-17

\footnotetext{
* Correspondencia del autor (Correo electrónico: dcarrillo@utleon.edu.mx)

$\dagger$ Investigador contribuyendo como primer autor
} 


\section{Introducción}

La cinemática del cuerpo humano ha sido sujeto de numerosas investigaciones a lo largo de la historia, principalmente en áreas como bioingeniería, biomédica, ciencias del deporte y animaciones en 3D [I]. En la actualidad uno de los desafíos que se presenta en el área de bioingeniería es el desarrollo de un modelo matemático y entorno grafico que emule el movimiento del cuerpo humano, es decir, que permita recrear, simular y analizar movimientos durante el ciclo de marcha de cualquier persona [II-III].

Actualmente existen desarrollos tecnológicos disponibles en el mercado que abarcan una gran cantidad de estas características de estudio acerca de la cinemática, principalmente sistemas ópticos área donde se reportan el mayor número de publicaciones, siendo la tecnología VICON la más utilizada, debido a que tiene una gran variedad de aplicaciones, sin embargo, es una tencnologia a la cual no todos pueden tener acceso debido a su alto costo. Por otra parte, en la actualidad en el país el número de pacientes amputados mantiene un incremento anual con más de 27 mil casos de amputaciones de miembros superiores e inferiores, las principales causas son: traumatismos, problemas congénitos o cáncer y vasculares [VI,V].

En los últimos años se han desarrollado equipos protésicos activos con la finalidad de mejorar la calidad de vida del paciente, sin embargo, debido a diversos factores como mecanismos pesados y ruidosos, tiempos de respuesta de los actuadores inadecuados y alineaciones ineficientes que provocan poca movilidad y limitan al paciente a adaptarse a un equipo protésico. Uno de los desafíos que se presenta en el desarrollo de las prótesis activas, es el diseño de un modelo matemático y entorno grafico que emule el movimiento del cuerpo humano, es decir, que permita recrear, simular y analizar movimientos durante el ciclo de marcha de cualquier persona. Esto con la intención de desarrollar mejores elementos como exoesqueletos, prótesis, órtesis, etc.

El objetivo del presente trabajo fue desarrollar una interfaz gráfica que permita simular el ciclo de marcha, obtener los rangos de movimiento y la velocidad angular en las rodillas y tobillos.

\section{Metodología}

El desarrollo de la interfaz gráfica fue con intención de tener una herramienta capaz de obtener un entorno grafico que emule el movimiento del cuerpo humano, es decir, que permita recrear, simular y analizar movimientos durante el ciclo de marcha de cualquier persona.

Se creó en el software Python 3.7, para su diseño se importaron las librerías tkinter, matplotlib, pandas. Para la creación de los menus fue necesario crear una base de datos .txt, estos datos serán utilizados en todos los apartados, cada uno de ellos es independiente no es necesario utilizar los menus en algún orden, sin embargo, se propone que se comience con el menu numero 1 para observar el movimiento que realiza la persona durante el estudio y asi proceder a interpretar los resultados de los siguientes menus.

A partir de de las coordenadas espaciales se ubican los tres marcadores de la zona del tobillo o de la zona de la rodilla y se determina la distancia $\mathrm{X}, \mathrm{Y}$ y $\mathrm{Z}$ entre los tres marcadores, con intención de formar un triangulo y por medio de la ley de cosenos determinar el angulo que genera el marcador.

Para determinar la velocidad angular en tobillos y rodillas fue necesario utilizar los angulos determinados en los movimientos angulares utilizando la siguiente ecuación:

$\omega=\frac{\Delta \theta}{\Delta t}$

Se realizó un estudio para determinar si había diferencia significativa entre el software de VICON y la interfaz, cabe mencionar que la base de datos es obtenida apartir del software de VICON.

El estudio consistió en comparar los rangos de movimiento generados en el software de VICON y los obtenidos en la interfaz. Para el caso de VICON, se obtuvo un promedio de $45.72^{\circ} \pm 1.45^{\circ}$ en la rodilla izquierda y $46.91^{\circ} \pm$ $0.34^{\circ}$ en la rodilla derecha. La interfaz obtuvo un promedio de $46.53^{\circ} \pm 1.17^{\circ}$ en la rodilla izquierda y $47.1^{\circ} \pm 0.52^{\circ}$ en la rodilla derecha. El coeficiente de variación para el software de VICON fue de $3.18 \%$ en rodilla izquierda y $0.73 \%$ en rodilla derecha y para la interfaz fue de $2.52 \%$ en rodilla izquierda y $1.12 \%$ en rodilla derecha. 
Finalmente, el análisis de los datos mostró que la diferencia de los resultados obtenidos entre la interfaz y VICON, no fue significativa, por lo que se puede concluir que la interfaz es una herramienta útil para el procesamiento de la información sin depender directamente del software VICON.

\section{Resultados}

La interfaz gráfica diseñada tiene cinco menus para la interpretación de datos, 1.- Adquisicion de datos, 2.- Análisis de rodillas, 3.- Análisis de tobillos, 4.-Velocidad angular de rodillas y 5.Velocidad angular de tobillos, el entorno de la interfaz consiste en el botón de salir, botón de soporte técnico y el fondo tiene la imagen de la Universidad Tecnológica de León, la Fig. 1 se muestra la estructura completa de la interfaz.

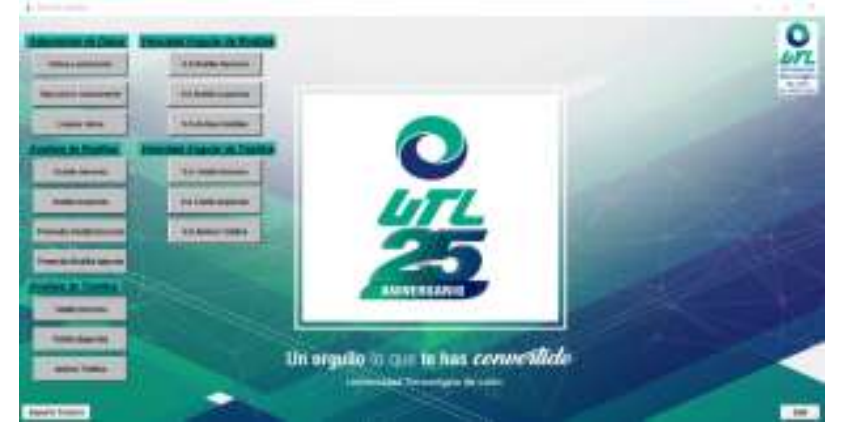

Figura 1 Diseño de la estructura de la interfaz Fuente: Elaboración Propia

\section{1.- Adquisicion de datos}

Este menú tiene 3 botones para obtener datos, reproducir animación y limpiar datos. La adquisición de datos consiste en abrir un archivo Excel con las coordenadas espaciales de las principales protuberancias oseas de los miembros inferiores del cuerpo humano, dichos marcadores se pueden ver en la figura 2 como círculos negros.

La opción de reproducir animación comienza la simulación del ciclo de marcha y finalmente el botón de limpiar datos permite borrar toda la información para seleccionar un nuevo caso de estudio.

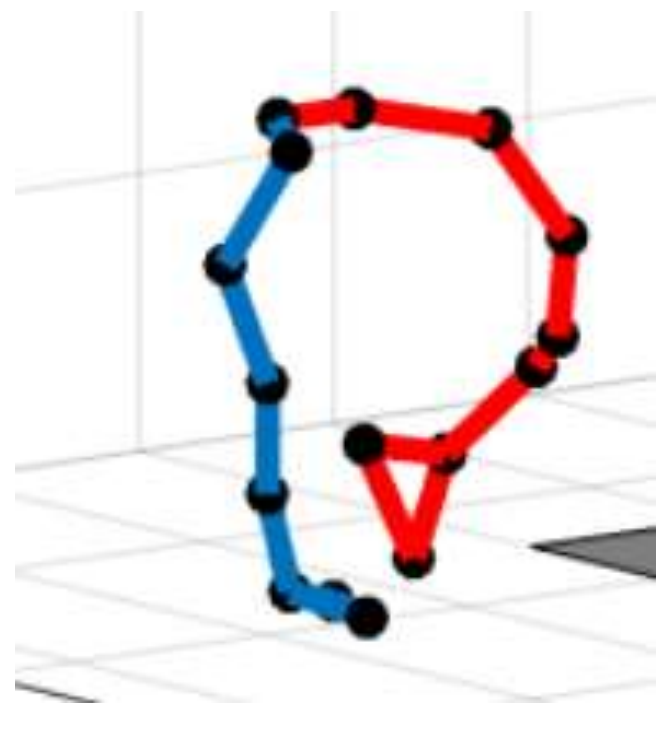

Figura 2 Simulación del ciclo de marcha Fuente: Elaboración Propia

\section{2.- Análisis de rodillas}

La información que se analiza en el menú anterior es interpretada para obtener los rangos de movimiento angular de la rodilla derecha e izquierda, se realiza apartir de 3 marcadores situados en dicha zona, el resultado lo podemos observar en la Fig. 3 cuyos valores corresponden a lo que se presenta en las investigaciones ya desarrolladas [VI-VII].

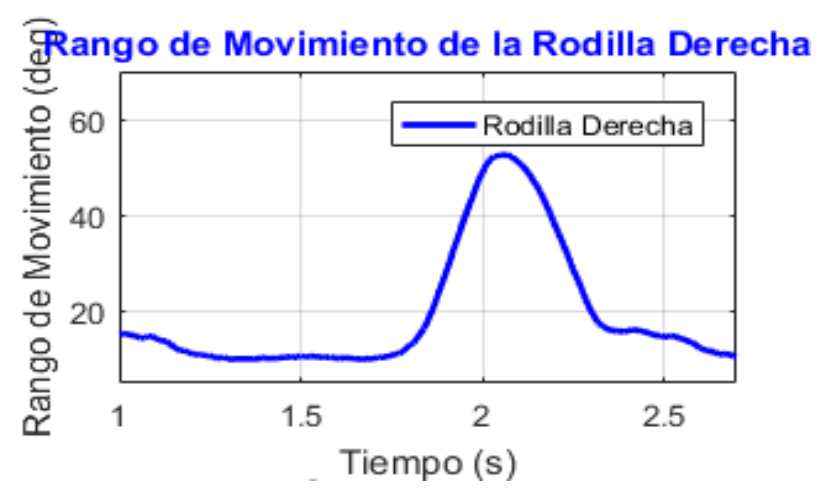

Figura 3 Rango de movimiento de la Rodilla Derecha Fuente: Elaboración Propia

Adicional se obtiene el promedio de movimiento de cada rodilla (línea azul) y la desviación estándar (líneas punteadas), esto se desarrolla a partir del numero total de ciclos de marcha completos que se realizan al obtener los datos, como se muestra en la siguiente figura 4. 


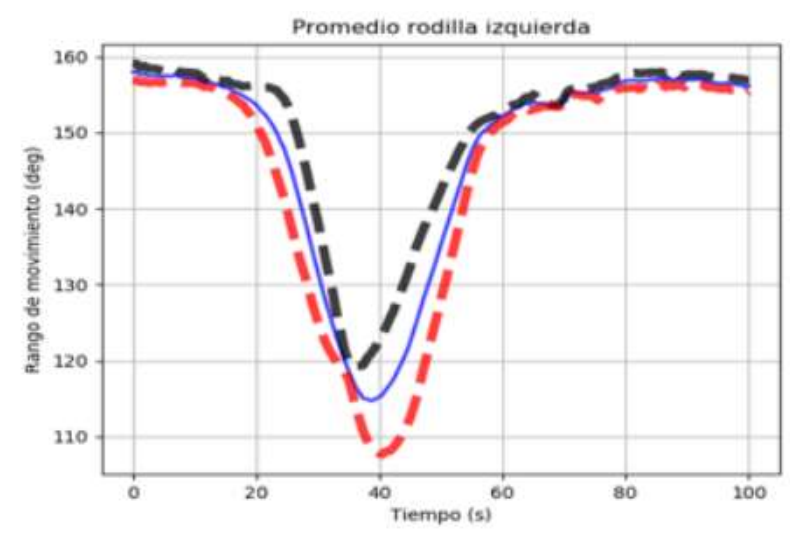

Figura 4 Promedio de la Rodilla Izquierda Fuente: Elaboración Propia

\section{3.- Análisis de tobillos}

De igual manera la información analizada en el menú 1, es interpretada para obtener el rango de movimiento de los tobillos, para ello se utilizan tres marcadores, en la siguiente figura se puede observar que los rangos de movimiento del tobillo corresponden a lo que se presenta en las investigaciones ya desarrolladas [VI-VII] (ver figura 5).

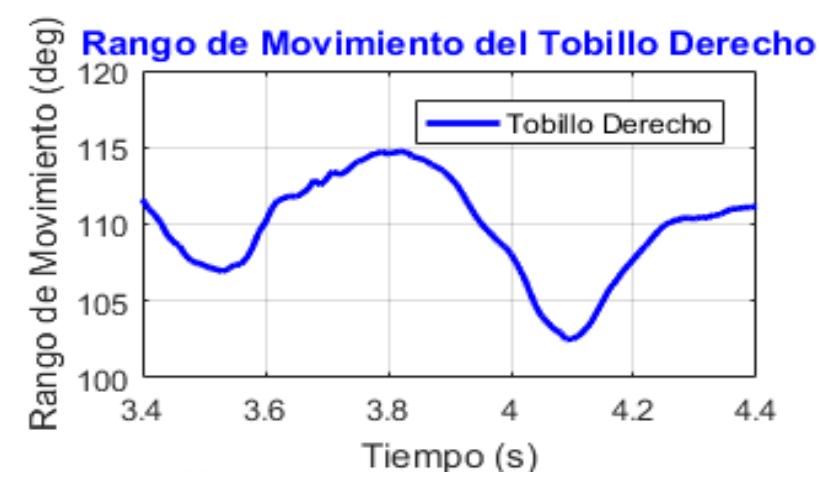

Figura 5 Rango de Movimiento del Tobillo Derecho Fuente: Elaboración Propia

\section{4.-Velocidad angular de rodillas}

Se determino a partir de los datos generados en el menú 2, en la Fig.6 se puede ver el resultado de la velocidad angular en función del tiempo.

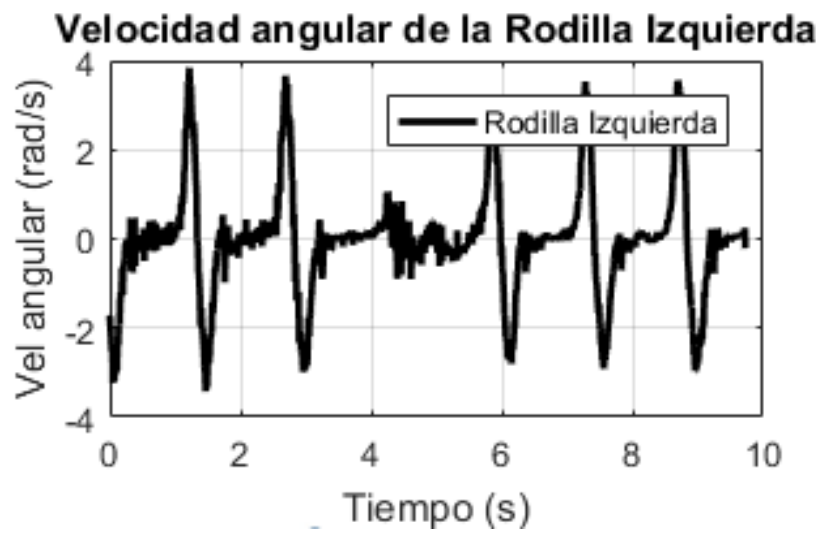

Figura 6 Velocidad angular de la Rodilla Izquierda Fuente: Elaboración Propia

\section{5.- Velocidad angular de tobillos}

La velocidad angular de los tobillos se determino a partir de la información obtenida en el menú 3, aplicando la ec.1, el resultado se puede observar en la figura 7.

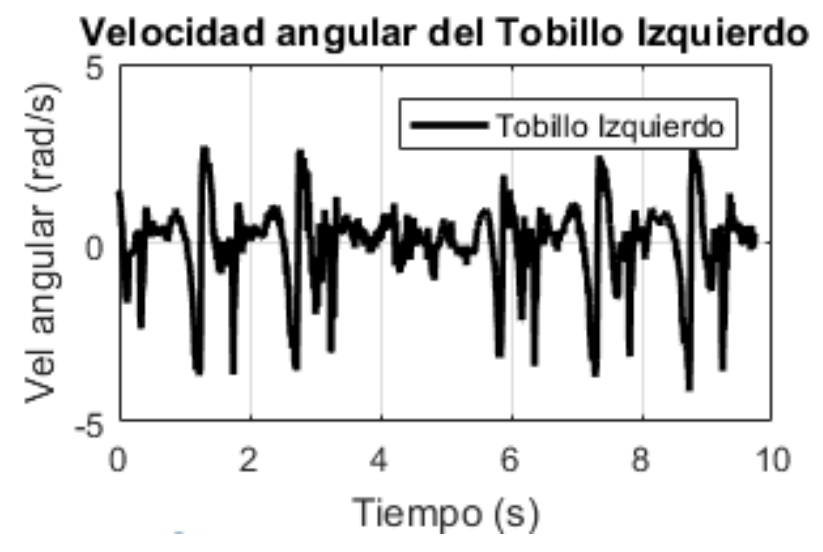

Figura 7 Velocidad angular del Ttobillo Izquierdo Fuente: Elaboración Propia

\section{Agradecimiento}

El principal agradecimiento es para la Universidad Tecnológica de León por otorgar el tiempo y equipo necesario para el desarrollo del proyecto, también se agradece a los colaboradores por su gran esfuerzo y tiempo invertido.

\section{Conclusiones}

En el presente trabajo se desarrolló una interfaz gráfica capaz de registrar y procesar la información del ciclo de marcha haciendo uso de software libre (Python 3.7). Dicha información fue previamente capturada por un sistema VICON el cual entrega las coordenadas espaciales de los puntos clave en un ciclo de marcha (rodillas y tobillos). 
Logrando realizar el procesamiento de información el cual funge como base para el cálculo de las velocidades angulares de las articulaciones inferiores, que a su vez entregan la información necesaria para la caracterización del ciclo de marcha de un paciente y lograr un mejor diseño de una prótesis.

Es de vital importancia reforzar el aprendizaje de un ámbito que crece cada día más y que toma gran valor a favor de la salud del humano, en este caso fisiológico.

Como futuro análisis se busca la obtención de la información en tiempo real con un sistema de visión propio y de bajo costo, lo cual contribuiría en extender el uso de esta tecnología y poder generar un mayor apoyo a quienes necesitan de una prótesis para el desarrollo de sus actividades diarias.

\section{Referencias}

[I] Martinez Villalpando, E. C., (2012). "Design and evaluation of a biomimetic agonist-atagonist knee prosthesis," p. 102.

[II] Contreras Bravo, L. E. and Roa Garzón, M. A. (2005), "Modelamiento de la marcha humana por medio de gráficos de unión". Tecnura, vol. 8, núm, 16, pp 26-42.

[III] Contreras, L. E., Tristancho, J.A. and Vargas, L. F. (2012). “Análisis Biomecánico de Marcha Humana a través de Técnicas de Modelaje", año 6, No. 12, pp 29-35.

[VI] Vazquez Vela, E., Hijar Medina, M., R. P. L, Espinosa Jove, I. G., and Rojas, X., (2015). "Los amputados, un reto para el Estado," Acta la Sesión la Acad. Nac. Med.

[V] Vázquez Vela, E., (2016). Los amputados y su rehabilitación.

[VI] Espinosa Gúzman, E. E. And García Vidal, U. O., (2015) "Prototipo de exoesqueleto electromecánico para complementar el ciclo de marcha humana en individuos con paraplejia,".

[VII] Garikayi, T., (2018). "Development of a robust myoelectric control architecture for lower limb robotic prosthetic applications,". 